\title{
Using research animals as sentinels
}

\begin{abstract}
Almost 20 years ago $^{1}$ and more recently in its Frequently Asked Questions ${ }^{2}$, the NIH's Office of Laboratory Animal Welfare (NIH/OLAW) clarified that the use of sentinel animals in a laboratory animal disease recognition and prevention program should be covered by an IACUCapproved protocol. The Great Eastern University IACUC had always assumed that the school's IACUC-approved sentinel animal protocol met the intent of NIH/ OLAW for helping to assure the well-being of animals and compliance with federal regulations. Furthermore, over the years, through many vivarium accreditation site visits and inspections by the veterinary medical officers of the USDA, no comments were ever made about Great Eastern's sentinel animal protocol.
\end{abstract}

Dr. Rhett Parks' guinea pigs arrived on a Monday, and after a few days of acclimatization to the vivarium, the assigned veterinary technician collected fresh feces and swabs of cells from the mucosal surface of the animals' cheeks. The samples were to be used to test for the presence of pathogen DNA by the polymerase chain reaction (PCR) method. The Great Eastern sentinel animal protocol specified that colony animals would be used for this testing because of the simplicity of the collection procedures and the greater likelihood of detecting pathogens from pooled samples taken directly from colony animals.

At the semiannual program review, the sentinel animal program was discussed, and one of the participants asked a simple question: if samples for PCR analysis are taken directly from a researcher's animal rather than from a sentinel animal, and if the animal must be handled in order to obtain the sample, should this activity be approved on the researcher's protocol or on the sentinel animal protocol? A simple question, perhaps, but is the answer simple? What is your opinion?

1. Potkay, S., Garnett, N.L., Miller, J.G., Pond, C.L. \& Doyle, D.J. Frequently Asked Questions about the Public Health Service Policy on Humane Care and Use of Laboratory Animals. Lab Anim. (NY) 24, 24-26 (1995).

2. Public Health Service. Policy on Humane Care and Use of Laboratory Animals-Frequently Asked Questions. Protocol Review, Question No. D.16. (US Department of Health and Human Services, Washington, DC, 2006, revised 2013).

\section{RESPONSE}

\section{Consider the purpose}

\section{Sridhar Samineni, DVM, MS, PhD, DACLAM \& Richard W. Ermel, DVM, MPVM, PhD, DACLAM}

Quarantine, animal biosecurity and disease surveillance are critical components of a comprehensive veterinary care program to detect and prevent the introduction of pathogens into animal research facilities, thereby enhancing the quality of research animals and minimizing the potential of confounding experimental variables. As part of the IACUC-approved sentinel animal protocol (animal health surveillance program), Great Eastern University's veterinary care staff collects feces and swab samples directly from research animals for diagnostic testing. However, a semiannual program review raised the question of whether these sample collection procedures should be listed and approved on the research protocol or on the sentinel animal protocol. To address this question, one should consider the purpose of the sample collection and the personnel who carry it out.

Both the Animal Welfare Act (AWA) ${ }^{1}$ and the Guide for the Care and Use of Laboratory Animals (the Guide) ${ }^{2}$ indicate that the attending veterinarian has responsibility for the health and wellbeing of all laboratory animals used at the institution and authority to ensure the provision of an appropriate veterinary care program. Specifically, the Guide ${ }^{2}$ states that an adequate veterinary care program is an essential part of an institutional animal care and use program, which includes assessment of animal well-being and effective management of a preventive medicine program. Preventive medicine programs should consist of various combinations of policies, procedures and equipment related to quarantine, animal biosecurity and animal health surveillance. Similarly, for species covered by the AWA ${ }^{1}$, adequate veterinary care must be provided and should include the use of appropriate methods to prevent, control, diagnose and treat diseases and injuries. Based on guidance from both the Guide ${ }^{2}$ and the AWA ${ }^{1}$, diagnostic sample collection activities on research animals for routine animal health surveillance and preventive medicine programs can be considered part of an adequate and comprehensive veterinary care program.

In addition, Great Eastern University veterinary technicians have the appropriate training and expertise to carry out the proposed activities (handling the research animals and collecting samples for the animal health surveillance program). Furthermore, these activities are not related to the research protocol, collected samples are not used in the research study, and animals are not handled by research staff for these procedures. Moreover, previous semiannual inspections and other regulatory authorities felt that the listing of these procedures in the IACUCapproved sentinel animal protocol was appropriate and justified. Therefore, Great Eastern University routine veterinary care 